\title{
A Practical Deep Learning Model in Differentiating Pneumonia-Type Lung Carcinoma from Pneumonia on CT Images: ResNet Added with Attention Mechanism
}

\author{
Wang Du, Xiaojie Luo, and Min Chen \\ Department of Radiology, Beijing Hospital, National Center of Gerontology, Institute of Geriatric Medicine, \\ Chinese Academy of Medical Science, Beijing 100730, China \\ Correspondence should be addressed to Min Chen; chenming9896@163.com
}

Received 26 November 2021; Revised 26 December 2021; Accepted 31 December 2021; Published 23 February 2022

Academic Editor: Wei long Zhong

Copyright (c) 2022 Wang Du et al. This is an open access article distributed under the Creative Commons Attribution License, which permits unrestricted use, distribution, and reproduction in any medium, provided the original work is properly cited.

Objective. We aim to develop a deep neural network model to differentiate pneumonia-type lung carcinoma from pneumonia based on chest CT scanning and evaluate its performance. Materials and Methods. We retrospectively analyzed 131 patients diagnosed with pneumonia-type lung carcinoma and 171 patients with pneumonia treated in Beijing Hospital from October 2019 to February 2021. The average age was $68( \pm 15)$ years old, and the proportion of men $(162 / 302)$ was slightly more than that of women (140/302). In this study, a deep learning based model UNet was applied to extract lesion areas from chest CT images. Lesion areas were extracted and classified by a designed spatial attention mechanism network. The model AUC and diagnostic accuracy were analyzed based on the results of the model. We analyzed the accuracy rate, sensitivity, and specificity and compared the results of the model to the junior and senior radiologists and radiologists based on the model. Results. The model has a good efficiency in detecting pneumonia-like lesions (6.31 seconds/case). The model accuracy rate, sensitivity, and specificity were $74.20 \%, 60.37 \%$, and $89.36 \%$, respectively. The junior radiologist's accuracy rate, sensitivity, and specificity were $61.00 \%, 48.08 \%$, and $75.00 \%$, respectively. The senior radiologist's accuracy rate, sensitivity, and specificity were $65.00 \%, 51.92 \%$, and $79.17 \%$, respectively. The results of junior radiologists based on the model were improved $(76.00 \%$ for accuracy rate, $62.75 \%$ for sensitivity, and $89.80 \%$ for specificity). The results of senior radiologists based on the model were also improved (78.00\% for accuracy rate, $64.71 \%$ for sensitivity, and $91.84 \%$ for specificity) and the diagnostic accuracy of which was statistically higher than other groups $(P<0.05)$. Based on the lesion texture diversity and the lesion boundary ambiguity, the algorithm produced false-positive samples (13.51\%). Conclusion. This deep learning model could detect pneumonia-type lung carcinoma and differentiate it from pneumonia accurately and efficiently.

\section{Introduction}

Lung cancer is the most common malignant tumor in the pulmonary disease, with about 1.8 million patients worldwide [1]. The incidence has increased significantly in the last 50 years [2]. Lung cancer is clinically divided into small cell lung cancer (SCLC) and nonsmall cell lung cancer (NSCLC), among which NSCLC accounts for $80 \%$. The most common histological subtypes of NSCLC are adenocarcinoma and squamous cell carcinoma (SQCC) [3]. However, in recent years, the incidence of pneumonic type lung carcinoma (PTLC) has been increasing, accounting for $0.48-3.33 \%$ of primary lung cancer, which is mainly seen in middle-aged and elderly patients, and the pathological type is adenocarcinoma $[4,5]$. Most patients with PTLC have a history of smoking, with the main manifestations of cough and sputum accompanied by fever. Most of the lesions are distributed around the lung. Laboratory and imaging examinations show no characteristic manifestations in the early stage of the disease, resulting in a high rate of misdiagnoses as pneumonia, tuberculosis, among other diseases in terms of symptoms and imaging manifestations at the first diagnosis [6-8]. 
TABLE 1: Summary of training and independent testing datasets.

\begin{tabular}{|c|c|c|c|c|c|c|}
\hline \multirow{3}{*}{ Parameter } & \multicolumn{2}{|l|}{ Training set } & \multicolumn{3}{|c|}{ Independent testing set } & \multirow{3}{*}{ Pneumonia } \\
\hline & PTLC & & Pneumonia & PTLC & & \\
\hline & \multicolumn{2}{|c|}{$P$ value } & & \multicolumn{2}{|c|}{$P$ value } & \\
\hline $\begin{array}{l}\text { No. of } \\
\text { patients }\end{array}$ & $88(43.6)$ & $114(56.4)$ & & $43(43)$ & $57(57)$ & \\
\hline Male patients & $40(19.8)$ & $68(33.7)$ & 0.550 & $27(27.0)$ & $33(33.0)$ & 0.468 \\
\hline Age $(y)^{*}$ & $68.23 \pm 12.46$ & $66.28 \pm 16.16$ & 0.347 & $69.42 \pm 16.54$ & $69.34 \pm 15.21$ & 0.424 \\
\hline $\begin{array}{l}\text { Pathology } \\
\text { examination }\end{array}$ & $\begin{array}{l}\text { Adenocarcinoma, } n=67 \text {; } \\
\text { squamous cell carcinomas, } \\
n=14 \text {; small cell } \\
\text { undifferentiated } \\
\text { carcinoma, } n=7\end{array}$ & $\begin{array}{c}\text { Bacterial } \\
\text { pneumonia, } \\
n=24 ; \text { viral } \\
\text { pneumonia, } \\
n=11 ; \text { CAP, } \\
n=79\end{array}$ & & $\begin{array}{l}\text { Adenocarcinoma, } n=33 ; \\
\text { squamous cell carcinomas, } \\
n=7 \text {; small cell } \\
\text { undifferentiated } \\
\text { carcinoma, } n=3\end{array}$ & $\begin{array}{c}\text { Bacterial } \\
\text { pneumonia, } \\
n=16 ; \text { viral } \\
\text { pneumonia, } n=7 ; \\
\text { CAP, } n=34\end{array}$ & \\
\hline
\end{tabular}

Values in parentheses are percentages. PTLC, pneumonic-type lung carcinoma; CAP, community-acquired pneumonia. ${ }^{*}$ Ages are reported as mean$\mathrm{s} \pm$ standard deviations.

Recently, computed tomography has been continuously developing. HRCT, the MPR function of MSCT, energy spectrum CT quantitative analysis, and dual-source CT dualenergy technology have been applied in the differential diagnosis of PTLC and pneumonia. They provided more information for CT diagnosis, thus enlarged the application scope of CT and improved diagnostic accuracy. Meanwhile, deep learning technology has achieved great success in medical imaging due to the powerful feature extraction ability [9-11]. Specifically, deep learning has been applied to children's chest radiograms to detect and identify bacterial and viral pneumonia $[12,13]$. There have also been attempts to detect various imaging features of chest CT $[14,15]$. At present, studies have applied deep learning technology for detecting and analyzing pneumonia lesions in CT images [16-18]. Other studies conducted an algorithmic analysis of lung cancer [19-21]. However, the current work mainly focuses on analyzing disease separately. There is a lack of study on the differentiation of multiple diseases, especially pneumonia and PTLC, which is very similar in imaging characteristics. Therefore, specific models were warranted to distinguish between the two diseases. The ResNet network uses cross-layer connections to strengthen the ability of gradient backpropagation, and attention mechanism is commonly used as an explicit feature selection operation in the field of computer vision. We used the RESNET neural network and added the attention mechanism to accurately locate the features in the network and extract the critical features for diagnosis. CT images of pneumonia and PTLC were included to test the stability of the model.

\section{Materials and Methods}

2.1. Patients. The institutional ethics committee approved this retrospective study. The need to obtain written informed consent for participation was waived.

Chest CT images from 302 patients from October 2019 to February 2021 were included in this study, comprising 171 patients with pneumonia and 131 patients with PTLC. Among them, there were 162 males and 140 females with an average age of 68 .
Inclusion criteria: patients with pneumonia-type lung cancer or pneumonia diagnosed by CT were included. The image quality of CT was good. Exclusion criteria: cases with poor image quality affecting observation.

All eligible patients underwent laboratory examination, routine pathological examination, or anti-inflammatory therapy.

The chest CT image with pneumonia and PTLC were randomly selected from October 2019 to February 2021 and randomly divided into a training set and a test set at a ratio of $2: 1$. The training dataset was then further subdivided into the training and internal validation (10\% of the sample). Independent test sets were not used for training and internal validation. The patient demographic and disease statistics are given in Tables 1 and 2, separately.

2.2. Imaging Protocol. The chest CT image was obtained with equipment from different manufacturers by using standard imaging protocols. Chest CT scan field from the thoracic inlet to the base of the lungs. Protocols are as follows: scanning slice thickness, $5 \mathrm{~mm}$; image matrix, $512 \times 512$; FOV, $400 \mathrm{~mm}$; reconstruction parameter setting: slice thickness, $1.0 \mathrm{~mm}$; layer spacing, $1.0 \mathrm{~mm}$; observation window parameters: lung window width, $1500 \mathrm{HU}$, window position, $-600 \mathrm{HU}$; mediastinum window width, $400 \mathrm{HU}$; window level, $35 \mathrm{HU}$.

2.3. Deep Learning Model. First, a segmentation model based on UNet [22] was designed (Figure 1). UNet employed feature extraction and reconstruction are based on multiscale information fusion. Specifically, the extraction model was a cascade of residual block [23] to extract CT image feature from different scales. Then, UNet performed feature reconstruction. When the feature scaled up, the features of previous reconstruction and corresponding scale in the downsampling process were merged to ensure the completeness of the information.

In the stage of lesion classification, we designed a classification network with the spatial attention mechanism (Figure 2). In the process of feature extraction, the attention mechanism was used to filter out unrelated features. The 
TABLE 2: Evaluation indexes of overall effectiveness of the radiologist, deep learning, and radiologist joint model in diagnosing pneumonialike lesions.

\begin{tabular}{|c|c|c|c|c|c|}
\hline & Junior radiologist & Senior radiologist & Model & $\begin{array}{c}\text { Junior } \\
\text { radiologist }+ \text { model } \\
\end{array}$ & $\begin{array}{c}\text { Senior } \\
\text { radiologist }+ \text { model } \\
\end{array}$ \\
\hline $\begin{array}{l}\text { No. of correct } \\
\text { diagnosis }\end{array}$ & $25 / 36$ & $27 / 38$ & $32 / 42$ & $32 / 44$ & $33 / 45$ \\
\hline LLF & $61 \%$ & $65 \%$ & $74 \%$ & $76 \%$ & $78 \%$ \\
\hline NLF & $\begin{array}{c}32.4 \% \\
\text { (95\% CI: } \\
0.19-0.50)\end{array}$ & $\begin{array}{c}27.01 \% \\
(95 \% \mathrm{CI}: \\
0.14-0.44)\end{array}$ & $\begin{array}{c}13.51 \% \\
(95 \% \mathrm{CI}: \\
0.05-0.30)\end{array}$ & $\begin{array}{c}13.51 \% \\
(95 \% \text { CI: } 0.05-0.30)\end{array}$ & $\begin{array}{c}10.81 \% \\
(95 \% \text { CI: } 0.04-0.26)\end{array}$ \\
\hline Sensitivity & $\begin{array}{c}48.0 \% \\
\text { (95\% CI: } \\
0.34-0.62)\end{array}$ & $\begin{array}{c}51.92 \% \\
(95 \% \mathrm{CI}: \\
0.38-0.66)\end{array}$ & $\begin{array}{c}60.37 \% \\
(95 \% \mathrm{CI}: \\
0.46-0.73)\end{array}$ & $\begin{array}{c}62.75 \% \\
(95 \% \text { CI: } 0.48-0.76)\end{array}$ & $\begin{array}{c}64.71 \% \\
(95 \% \text { CI: } 0.50-0.77)\end{array}$ \\
\hline Specificity & $\begin{array}{c}75.0 \% \\
(95 \% \mathrm{CI}: \\
0.60-0.86)\end{array}$ & $\begin{array}{c}79.17 \% \\
\text { (95\% CI: } \\
0.65-0.89)\end{array}$ & $\begin{array}{c}89.36 \% \\
(95 \% \mathrm{CI}: \\
0.76-0.96)\end{array}$ & $\begin{array}{c}89.80 \% \\
(95 \% \text { CI: } 0.77-0.96)\end{array}$ & $\begin{array}{c}91.84 \% \\
(95 \% \text { CI: } 0.80-0.97)\end{array}$ \\
\hline PPV & $\begin{array}{c}67.5 \% \\
(95 \% \mathrm{CI}: \\
0.50-0.81)\end{array}$ & $\begin{array}{c}72.97 \% \\
\text { (95\% CI: } \\
0.56-0.86)\end{array}$ & $\begin{array}{c}86.49 \% \\
(95 \% \mathrm{CI}: \\
0.70-0.95)\end{array}$ & $\begin{array}{c}86.49 \% \\
(95 \% \text { CI: } 0.70-0.95)\end{array}$ & $\begin{array}{c}89.19 \% \\
(95 \% \text { CI: } 0.74-0.96)\end{array}$ \\
\hline NPV & $\begin{array}{c}57.1 \% \\
(95 \% \text { CI: } \\
0.44-0.69) \\
\end{array}$ & $\begin{array}{c}60.31 \% \\
\text { (95\% CI: } \\
0.47-0.72) \\
\end{array}$ & $\begin{array}{c}66.67 \% \\
(95 \% \mathrm{CI}: \\
0.54-0.78) \\
\end{array}$ & $\begin{array}{c}69.84 \% \\
(95 \% \text { CI: } 0.57-0.80)\end{array}$ & $\begin{array}{c}71.43 \% \\
(95 \% \text { CI: } 0.58-0.82)\end{array}$ \\
\hline
\end{tabular}

spatial attention network is a ResNet network [23]. Before each feature downsampling, the features were fed to a convolution module with a constant output channel of 1 , and the sigmoid function was applied to the output to generate a weighted mask with the same width as feature. The value of each element in the weight mask ranged from 0 to 1 , which was multiplied by the feature to complete the feature filtering. The UNet model achieved the effect of state of the art in the field of semantic segmentation due to its structure of multilevel feature reconstruction and reuse of downsampling features. Meanwhile, UNet's multilevel feature multiplexing in the process of feature encoding greatly reduced the information loss in the network information transmission. Thus, the network had a better segmentation effect in difficult areas such as boundaries.

The attention mechanism made the network focus on important parts of the image during feature extraction. The information contained in the image was rich and diverse. The indiscriminate reference of all the information in the image would make the features complicated and confusing. The attention mechanism ensured that only the key features in the image were extracted by assigning different weights to the image features of different regions, resulting in decreased cost of network convergence and improved accuracy.

\subsection{Detection of Pneumonia-Like Lesions}

2.4.1. Artificial Diagnosis. A junior radiologist with 3-5 years of work experience and a senior chest imaging specialist with more than ten years of work experience independently read out the images without referring to the deep learning model. The location of pneumonia-like lesions was marked; the lesion features were selected; the qualitative diagnosis was made; the number of image layers was recorded based on the location of the lesions.
2.4.2. The Deep Learning Model Detected Pneumonia-Like Lesions. The chest CT images of 100 patients were imported into the model for automatic recognition, labeling, and diagnosis.

2.4.3. Deep Learning Models Assisted Radiologists in Diagnosis. Two weeks after the radiologist completed the independent readout of the images, the junior and senior radiologists used the deep learning model to assist the reading and recorded the diagnostic accuracy of pneumonialike lesions detected.

2.4.4. Reference Criteria for Qualitative Diagnosis of Pneumonia-Like Lesions. Two chest radiologists with more than ten years of experience, respectively, marked the chest CT images of 302 patients. The diagnostic results of the lesion were identified and recorded. The consensual results were combined with the results of pathology and laboratory tests as the reference standard for genuine positivity.

2.5. Statistical Analysis. SPSS 20.0 (IBM Corp., Armonk, NY, USA) was used to input, organize, and analyze the data. Levels of significant are shown as $P<0.05$.

(1) The performance of our trained deep learning model was evaluated using an independent test set that was not involved in the training process. The test set consisted of 100 examination images, which were randomly selected from 302 data. Unrestricted response receiver operating characteristic (ROC) curve was used to analyze and test the performance of the deep learning model to comprehensively reflect the performance of the model in terms of false-positive rate and detection rate. The area 


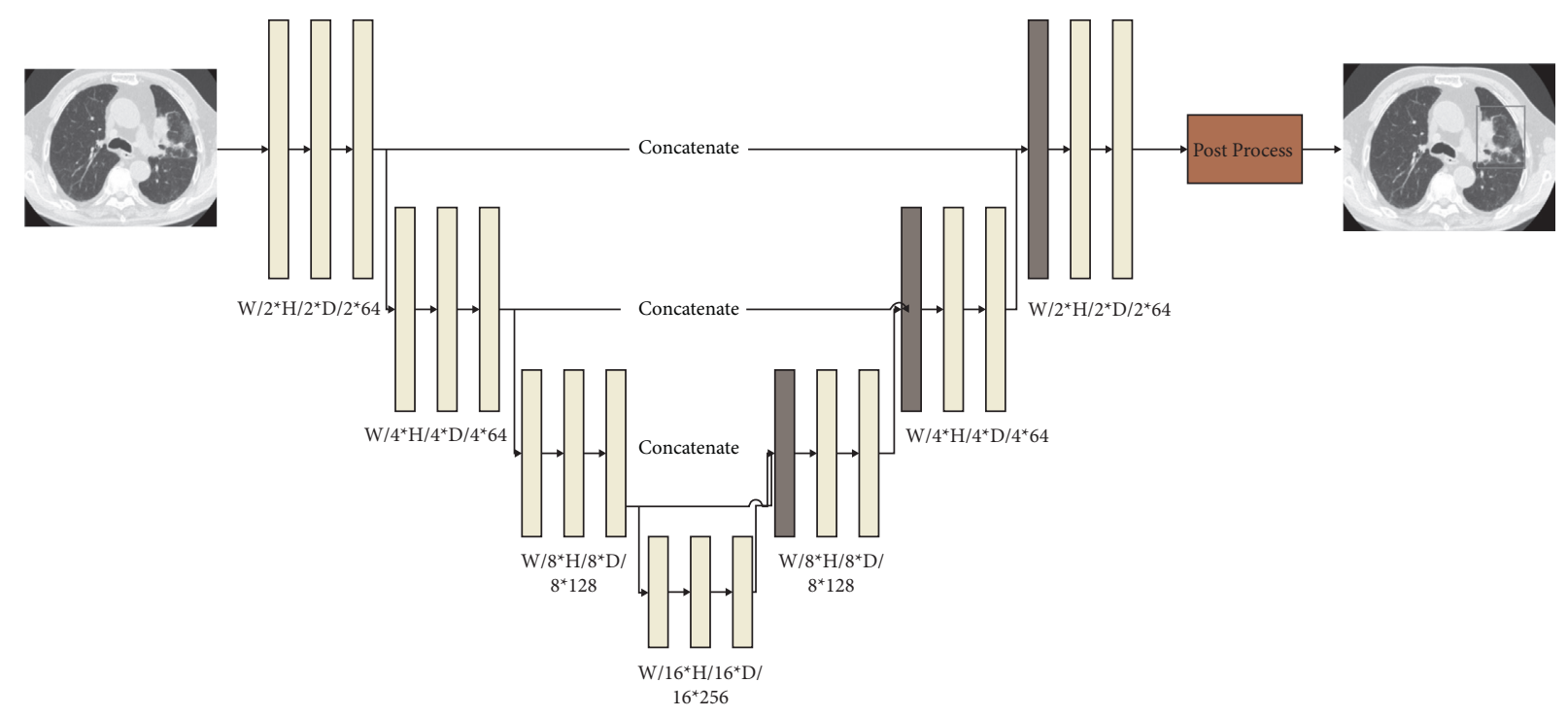

3D Convolution

Feature Merge +3 D Convolution

FIgURE 1: Segmentation model based on UNet.

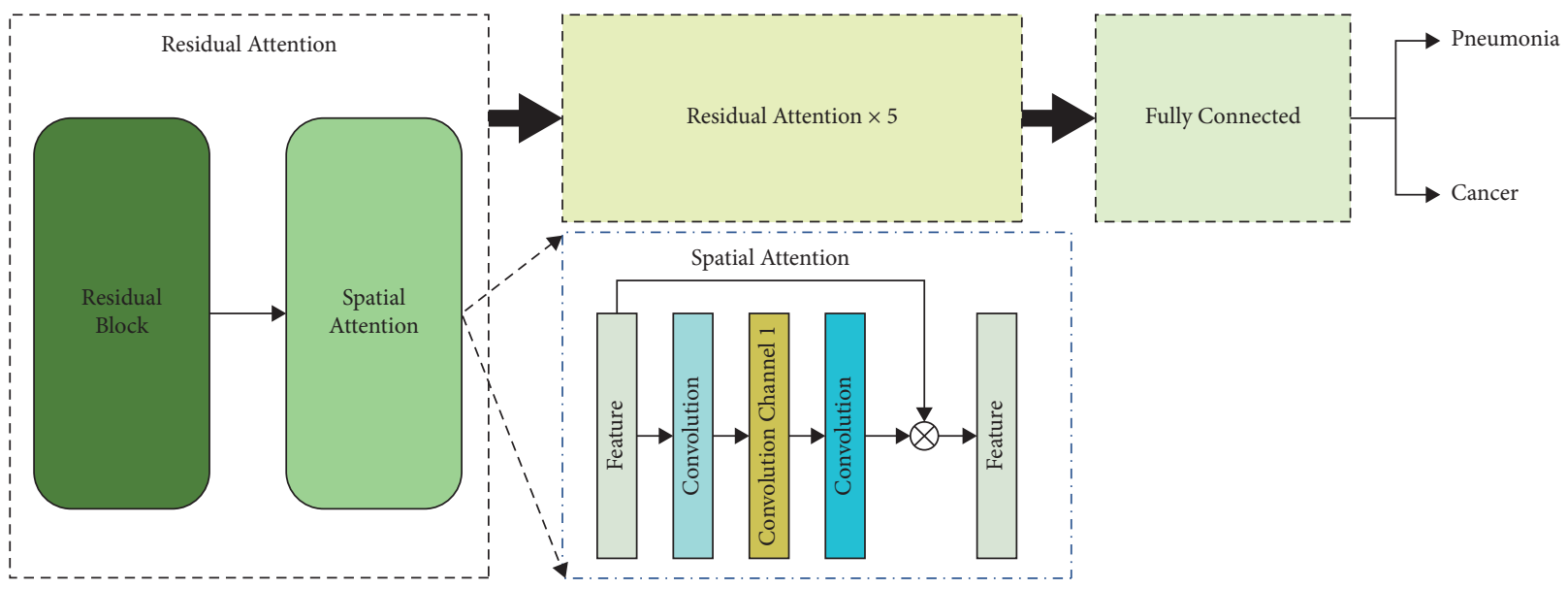

FIgURE 2: Architecture for the lesion classification.

under the curve was calculated using a $95 \%$ confidence interval (CI).

(2) With the assistance of the deep learning model, the differences in the diagnostic accuracy of pneumonia-like lesions among the five groups were compared among the doctors with low seniority, the doctors with high seniority, the deep learning model, and the combined deep learning model of the doctors with high and low seniority, respectively. The chi-square test was used to make pairwise comparison between groups.

\section{Results}

3.1. Study Population Characteristics. Table 1 provides the demographic characteristics for the training and independent testing datasets. There were slightly more male than female patients across PTLC and pneumonia groups for both the training dataset (percentage of male patients: PTLC $19.8 \%$; pneumonia, 33.7\%; $P=0.55$ ) and the independent testing dataset (percentage of male patients: PTLC 27\%; pneumonia, 33\%; $P=0.468$ ). There was no significant difference in age between the two groups.

3.2. Deep Learning Model Performance. Once the model was trained, it was deployed on a server with NVIDIA 2080Ti graphics card, 16 GB RAM, Intel Xeon E5-2620 v4 @2.10 GHz. Inference speed of the model on this platform was fast, 6.25 seconds per study on average. The detailed performance of the model is shown in figure. An average AUC value of $0.82(P<0.01)$ (Figure 3$)$ was obtained via five-fold cross-validation. In the validation set, 74 cases were correctly diagnosed with pneumonia-like lesions and 26 cases were diagnosed incorrectly (Figures 4 and 5). The 


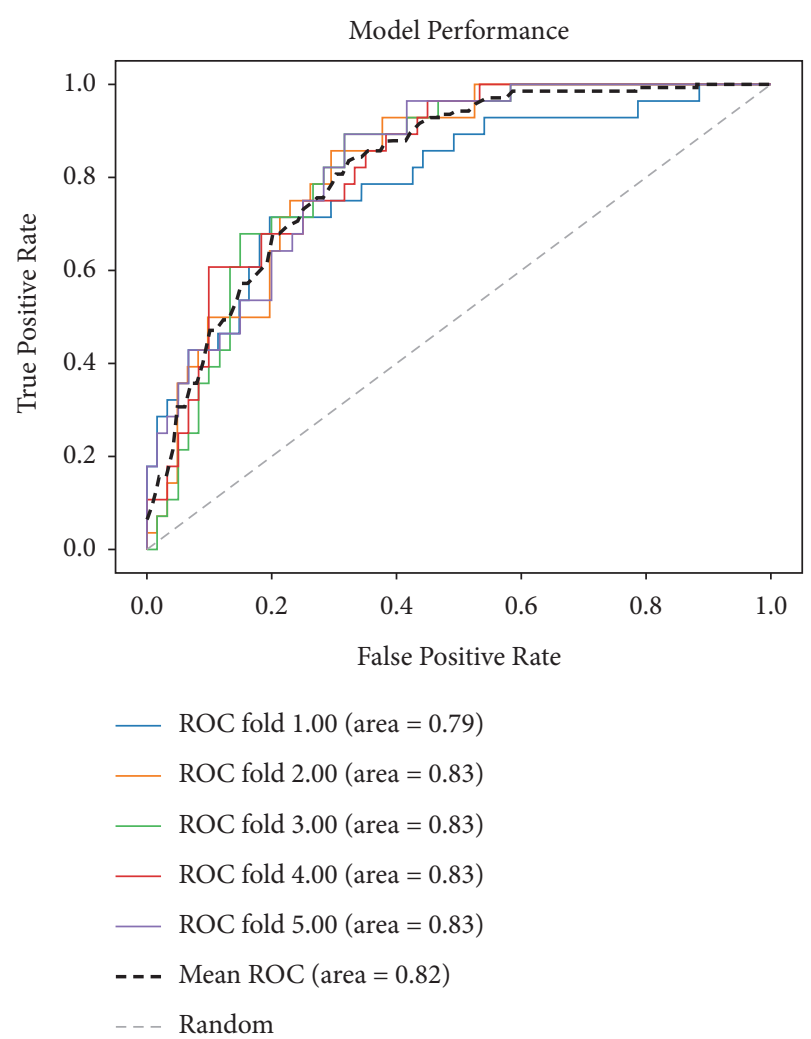

FIGURE 3: 5-fold validation ROC curves: the black dash line means the average of 5 curves, and the gray dash line means a model without any predictive ability. The average AUC value of FROC was $0.82(P<0.01)$ after five-fold cross-validation. The average accuracy of cross-validation was $74.2 \%$. The model introduced false-positive in the process of distinguishing pneumonia and lung cancer, but the overall accuracy had a relatively strong reference value. It shows that the model has high credibility.

average accuracy of five-fold cross-validation was $74.2 \%$ (Table 2). The model introduces false-positives in the process of distinguishing PTLC from pneumonia. The overall accuracy rate has a relatively strong reference value, but some cases seemed to be very challenging cases. In order to further improve the accuracy, more data might be required for multiple training.

\subsection{Comparison of Diagnostic Accuracy between the Deep Learning Model and Radiologists for Pneumonia-Like Lesions}

(i) The details of junior radiologists, senior radiologists, deep learning model, and radiologist based on the model of overall reading pneumonia sample number of correct diagnosis, correct diagnosis score (lesion localization fraction, LLF), false-positive diagnostic score (nonlesion localization fraction, NLF), sensitivity, specific, positive predictive value, and negative predictive value are given in Table 2 .

(ii) Comparison of diagnostic accuracy of pneumonialike disease among junior radiologists, senior radiologists, model, and radiologist based on model (Figure 6). The diagnostic accuracy of senior radiologists based on the model was the highest, which was statistically different from other groups $(P<0.05)$. The corresponding diagnostic accuracy rates of each group were $61 \%, 65 \%, 74 \%, 76 \%$, and $78 \%$, respectively, as given in Table 2 . Results of pairwise comparison between groups are given in Table 2 . There were no statistically significant differences in the diagnostic accuracy between any two following groups, including senior and junior radiologists $(P=0.558)$, model and senior radiologists $(P=0.167)$, model and senior radiologist based on the model $(P=0.774)$, model and junior radiologist based on model $(P=0.508)$, and junior radiologist based on model and senior radiologist $(P=0.088)$. There were statistically significant differences in the diagnostic accuracy between any two following groups, including the model and the junior radiologist, the junior radiologist based on the model and the junior radiologist, the senior radiologist based on the model and the junior radiologist, and the senior radiologist based on model and the senior radiologist (all $P<0.05$ ).

\section{Discussion}

PTLC is a special manifestation of peripheral lung cancer, which mainly manifested as lung lobe, segment, and ground glass density in imaging finding. The clinical manifestations are cough, expectoration, fever, and other symptoms, lack of specificity, with similar imaging to "pneumonia," which can be easily misdiagnosed as pneumonia and other diseases. As a result, patients cannot receive effective treatment in the first time, directly threatening the follow-up treatment and life [22-24]. Clinically, most patients with PTLC have pneumonia-like imaging changes [25], and mucinous cell type mucinous adenocarcinoma is the main pathological type [26]. In the past, PTLC was often assessed according to the imaging manifestations of bronchus accompanied by branch vessels, and it could be considered as PTLC in the case of pneumonia accompanied by irregular deformation and stenosis of bronchial lumen. Misdiagnosis or missed diagnosis often occurred in conventional CT examination [27].

In recent years, imaging technology in China has developed rapidly, and high-precision CT and PET/CT have become increasingly popular, which is of great help to distinguish between PTLC with different pathological basis and focal pneumonia. In a study, patients with pneumoniatype lung cancer received chest-enhanced CT, single-phase $\mathrm{PET} / \mathrm{CT}$, and dual-phase 18F-FDG PET/CT, respectively. The study found that chest enhanced CT combined with dual-phase F-FDG PET/CT had the highest diagnostic accuracy $(91.1 \%)$. The accuracy of chest enhanced CT alone was the lowest $(27.4 \%)$, proving that chest enhanced CT combined with dual-phase 18F-FDG PET/CT has an exact diagnostic effect. However, it is still misdiagnosed to a certain extent to assess benign and malignant lesions based 


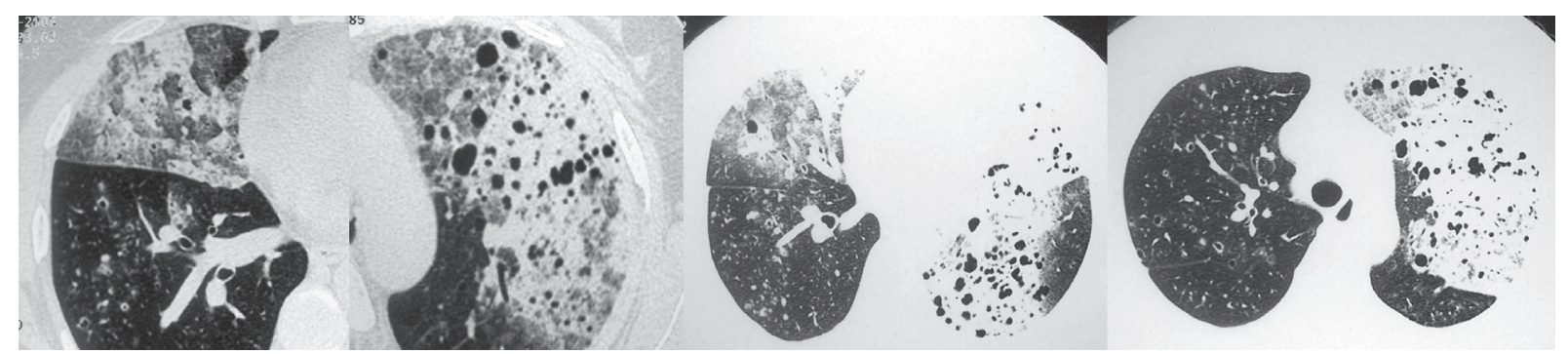

FIGURE 4: Representative example of a case of pneumonia-type lung carcinoma misclassified as pneumonia. The different slices around the abnormality are shown.

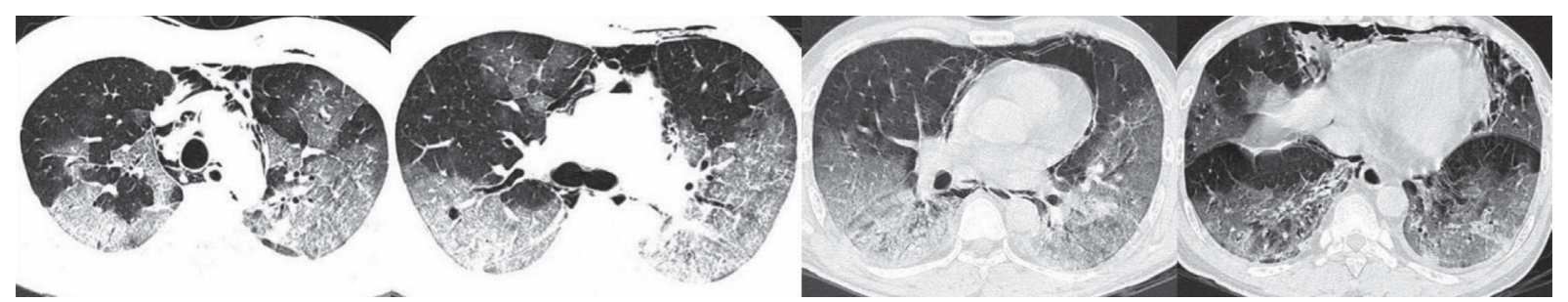

Figure 5: Representative example of a case of pneumonia misclassified as pneumonia-type lung carcinoma. The different slices around the abnormality are shown.

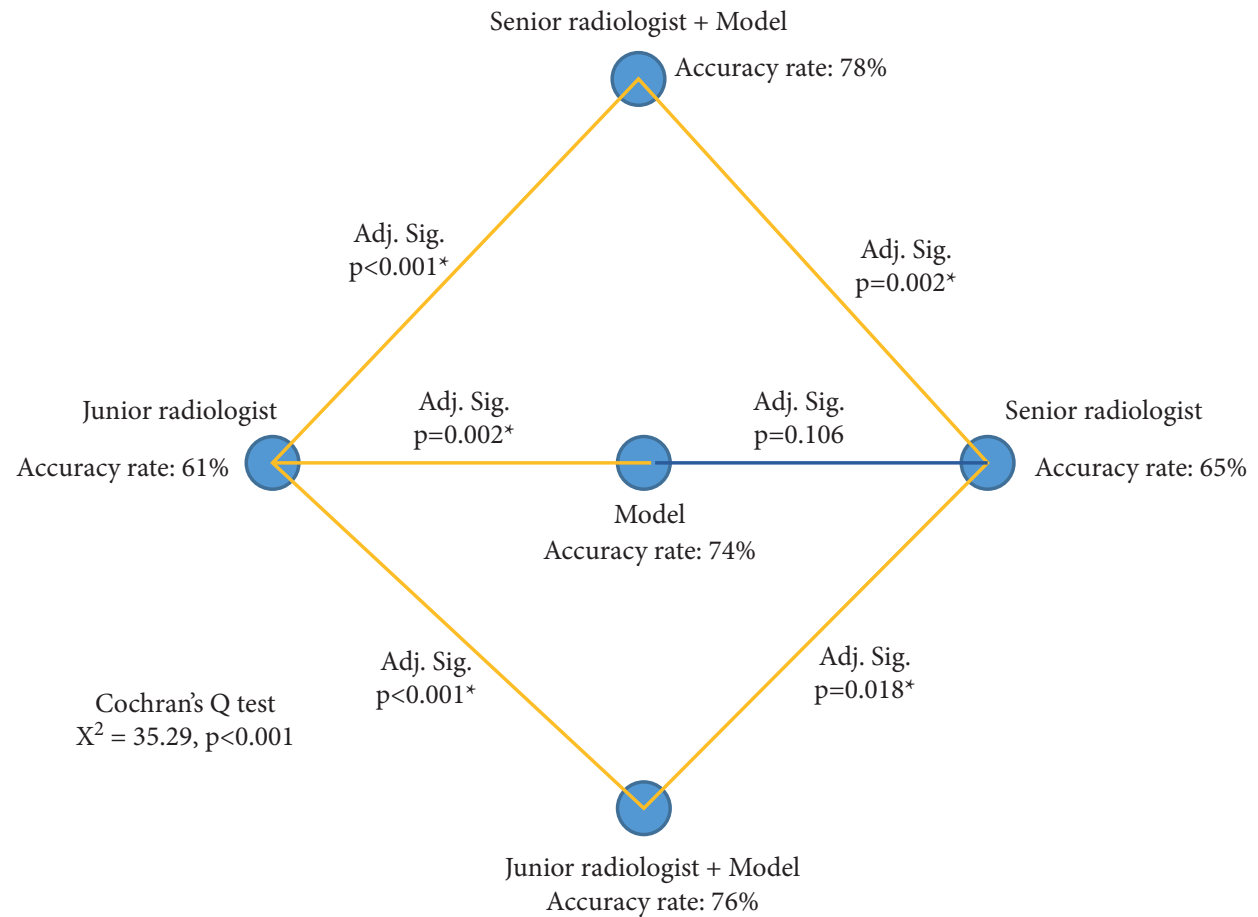

FiguRE 6: Comparison of overall diagnostic accuracy of pneumonia-like lesions among junior radiologist, senior radiologist, model, and radiologist joint model. Adj. Sig, adjusted significance. ${ }^{*}$ Variables significantly different $(P<0.05)$.

on the 18F-FDG glucose metabolism [28]. This examination will increase the patient's economic burden and radiation dose. In the past, when distinguishing PTLC from pneumonia, biopsy and pathology were used in addition to conventional CT examination, so as to promote the improvement of the diagnosis effect of PTLC. When using fibrobronchoscopy histological biopsy, the pathological diagnosis can be carried out under the guidance of CT, which can not only accurately judge the benign and malignant tumor but also clearly classify the tumor, providing a good diagnostic basis for subsequent treatment. The PTLC lacks the irregular mass and burr signs common to lung cancer on imaging, but is actually composed of numerous cancerous nodules without inflammatory lesions. The 
pathological basis may be due to the invasive development of cancer tissue itself in bronchus and alveoli, which covers the surface of alveolar wall when spreading in the airway and leads to consolidation of lung lobe in the process of mucus secretion [29]. However, there are some limitations in the puncture biopsy. Due to the influence of small biopsy specimens and deviation of sample acquisition site, there are sometimes missed diagnosis and misdiagnosis. Moreover, puncture biopsy is an invasive examination, which will bring psychological burden to patients.

With the rise of machine learning in recent years, some works have been focused on the field of pneumonia image processing. For example, a convolutional neural network was used to distinguish between pneumonia patients and healthy controls [30]. Besides, novel coronavirus pneumonia was differentiated from general pneumonia using the convolutional neural network [31]. Moreover, a variety of deep learning feature embedding methods were used to improve the recognition accuracy of COVID-19 pneumonia [32], screen diseased frames from pneumonia images [33], and screen patients with pneumonia [34]. However, there is little work in the field of distinguishing pneumonia and lung cancer. UNet achieves excellent results in the field of medical image segmentation because of its excellent feature coding structure [35-38]. The ResNet network uses cross-layer connections to strengthen the ability of gradient backpropagation, which is regarded as the best backbone network in many image processing tasks [39-41]. Attention Mechanism, as a commonly used explicit feature selection operation in the field of computer vision, affects the development of many machine learning tasks [42-45]. We have proposed a new model for the identification of PTLC and pneumonia and achieved good results via the combination of these technologies. In this study, we designed and evaluated a deep learning model to differentiate PTLC and pneumonia from the chest CT image. On an independent testing dataset, we showed that this model has high sensitivity $(60.37 \%)$ and specificity $(89.36 \%)$ in the detection of PTLC and pneumonia. The areas under the receiver operating characteristic curves for the model was 0.82 , suggesting good performance and high credibility. In terms of diagnostic accuracy, the combined model of senior radiologists was the highest $(78 \%)$. The model was higher than the manual model of junior radiologists and had no significant difference with the combined model of radiologists. This study showed that the sensitivity of the diagnosis increased from $48.08 \%$ to $64.71 \%$, the specificity increased from $75.00 \%$ to $91.84 \%$, and the false-positive rate decreased from $32.43 \%$ to $10.81 \%$ after the radiologists used the model. Therefore, the application of the model improved the diagnostic accuracy and efficiency.

However, this study has some limitations. There is a lot of overlap in the response of the lungs to various injuries, as well as in the presentation of many lung diseases. There is no way to distinguish all lung diseases based solely on the appearance of the chest CT image. At present, it is generally believed at home and abroad that the final results of computer-aided diagnosis should still be confirmed by the radiologists, so we suggest a multidisciplinary approach. In addition, although a large amount of data were collected for this study, the test set and the training set came from the same hospital. Finally, there is still room for improvement in the encoding of features. The feature extraction method based on convolution cannot pay attention to the global feature distribution of the lesion. The transformer [46] structure commonly used in the ViT field can be used to enhance the feature encoding to strengthen the attention of the feature to the global information of the lesion.

In conclusion, a machine learning method based on the convolutional network model was used to distinguish PTLC and pneumonia in this study. The results showed that the model has good performance and high reliability, and the diagnostic accuracy is significantly higher than simple chest CT. Meanwhile, compared with traditional examination methods, this method reduce radiation, economic, and psychological burden for patients. Therefore, this method has prospect for clinical application. However, this model has some deficiencies. In the future, we will collect more cases, increase the sample size, subdivide the pathological types, optimize the model, and apply the updated algorithm. We hope to develop a mature deep learning model to differentiate PTLC and pneumonia from the chest CT image more accurately and provide reference for treatment.

\section{Data Availability}

The analyzed datasets generated during the study are available from the corresponding author upon request.

\section{Conflicts of Interest}

The authors declare that they have no conflicts of interest.

\section{References}

[1] R. L. Siegel, K. D. Miller, and A. Jemal, "Cancer statistics, 2017," CA: A Cancer Journal for Clinicians, vol. 67, no. 1, pp. 7-30, 2017.

[2] L. A. Torre, F. Bray, R. L. Siegel, J. Ferlay, J. Lortet-Tieulent, and A. Jemal, "Global cancer statistics, 2012," CA: A Cancer Journal for Clinicians, vol. 65, no. 2, pp. 87-108, 2015.

[3] W. Chen, R. Zheng, P. D. Baade et al., "Cancer statistics in China, 2015," CA: A Cancer Journal for Clinicians, vol. 66, no. 2, pp. 115-132, 2016.

[4] A. Jemal, R. Siegel, J. Xu, and E. Ward, "Cancer statistics, 2010," CA: A Cancer Journal for Clinicians, vol. 60, no. 5, pp. 277-300, 2010.

[5] W. D. Travis, "Pathology of lung cancer," Clinics in Chest Medicine, vol. 32, no. 4, pp. 669-692, 2011.

[6] S. Iqbal, K. Iqbal, F. Arif, A. Shaukat, and A. Khanum, "Potential lung nodules identification for characterization by variable multistep threshold and shape indices from CT images," Computational and Mathematical Methods in Medicine, vol. 2014, no. 14, 7 pages, Article ID 241647, 2014.

[7] X. Gao, C. Chu, Y. Li et al., "The method and efficacy of support vector machine classifiers based on texture features and multi-resolution histogram from 18F-FDG PET-CT images for the evaluation of mediastinal lymph nodes in patients with lung cancer," European Journal of Radiology, vol. 84, no. 2, pp. 312-317, 2015. 
[8] M. M. Dua, J. M. Cloyd, F. Haddad, R. E. Beygui, J. A. Norton, and B. C. Visser, "Cardiac metastases and tumor embolization: a rare sequelae of primary undifferentiated liver sarcoma," International Journal of Surgery Case Reports, vol. 5, no. 12, pp. 927-931, 2014.

[9] D. Shen, T. Liu, T. M. Peters et al., Eds., in Proceedings of the 22nd International Conference on Medical Image Computing and Computer Assisted Intervention, vol. 11765, pp. 577-585, Springer, Shenzhen, China, October 2019, Lecture Notes in Computer Science.

[10] B. Kong, X. Wang, J. Bai et al., "Learning tree-structured representation for 3D coronary artery segmentation," Computerized Medical Imaging and Graphics, vol. 80, Article ID 101688, 2020.

[11] H. Ye, F. Gao, Y. Yin et al., "Precise diagnosis of intracranial hemorrhage and subtypes using a three-dimensional joint convolutional and recurrent neural network," European Radiology, vol. 29, no. 11, pp. 6191-6201, 2019.

[12] D. S. Kermany, M. Goldbaum, W. Cai et al., "Identifying medical diagnoses and treatable diseases by image-based deep learning," Cell, vol. 172, no. 5, pp. 1122-1131, 2018.

[13] S. Rajaraman, S. Candemir, I. Kim, G. Thoma, and S. Antani, "Visualization and interpretation of convolutional neural network predictions in detecting pneumonia in pediatric chest radiographs," Applied Sciences, vol. 8, no. 10, p. 1715, 2018.

[14] A. Depeursinge, A. S. Chin, A. N. Leung et al., "Automated classification of usual interstitial pneumonia using regional volumetric texture analysis in high-resolution computed tomography," Investigative Radiology, vol. 50, no. 4, pp. 261-267, 2015.

[15] M. Anthimopoulos, S. Christodoulidis, L. Ebner, A. Christe, and S. Mougiakakou, "Lung pattern classification for interstitial lung diseases using a deep convolutional neural network," IEEE Transactions on Medical Imaging, vol. 35, no. 5, pp. 1207-1216, 2016.

[16] M. Chumbita, C. Cillóniz, P. Puerta-Alcalde et al., "Can artificial intelligence improve the management of pneumonia," Journal of Clinical Medicine, vol. 9, no. 1, p. 248, 2020.

[17] K. Zhang, X. Liu, J. Shen et al., "Clinically applicable AI system for accurate diagnosis, quantitative measurements, and prognosis of COVID-19 pneumonia using computed tomography," Cell, vol. 181, no. 6, pp. 1423-1433, Article ID e11, 2020.

[18] G. Chassagnon, M. Vakalopoulou, E. Battistella et al., "AIdriven quantification, staging and outcome prediction of COVID-19 pneumonia," Medical Image Analysis, vol. 67, Article ID 101860, 2021.

[19] W. Sun, B. Zheng, and W. Qian, "Computer aided lung cancer diagnosis with deep learning algorithms," in Proceedings of the Medical imaging 2016: computer-aided diagnosis, vol. 9785, March 2016.

[20] N. Coudray, P. S. Ocampo, T. Sakellaropoulos et al., "Classification and mutation prediction from non-small cell lung cancer histopathology images using deep learning," Nature Medicine, vol. 24, no. 10, pp. 1559-1567, 2018.

[21] D. Ardila, A. P. Kiraly, S. Bharadwaj et al., "End-to-end lung cancer screening with three-dimensional deep learning on low-dose chest computed tomography," Nature Medicine, vol. 25, no. 6, pp. 954-961, 2019.

[22] O. Ronneberger, P. Fischer, and T. Brox, "U-net: convolutional networks for biomedical image segmentation," in Proceedings of the International Conference on Medical Image
Computing and Computer Assisted InterventionSpringer, Cham, October 2015.

[23] J. E. Cantera, M. P. Alfaro, D. C. Rafart, R. Zalazar, M. M. Muruzabal, P. G. Barquín, and I. V. Pérez, Inflammatory myofibroblastic tumours :a pictorial review," Insights Imaging, vol. 18, no. 8, pp. 8-9, 2014.

[24] L. Khorashadi, C. C. Wu, S. L. Betancourt, and B. W. Carter, "Idiopathic pulmonary haemosiderosis: spectrum of thoracic imaging findings in the adultpatient," Clinical Radiology, vol. 13, no. 1, pp. 6-9, 2014.

[25] G. Xu, "The experience of diagnosis and treatment of 10 cases with pneumonic-tupe lung Cancer CHSM," China Health Standard Management, vol. 9, no. 8, pp. 31-33, 2018.

[26] J. Zhao, L. Tong, Y. Fang, W. Zhang, M. A. Wanhui, and Y. Zhang, "CT diagnosis and differential diagnosis of pneumonia-type lung cancer," Medical Journal of the Chinese People's Armed Police Forces, vol. 28, no. 12, pp. 1236-1239, 2017.

[27] J. Zhao, W. Zhang, C. Fan, M. A. Wanhui, Y. Zhang, and L. I. Haitao, "Diagnostic value of two-phase PET-CT combined with enhanced CT for pneumonia type carcinoma of lung," Journal of third military medical university, vol. 40, no. 13 , pp. 1242-1246, 2018.

[28] Y. Liu, J. Li, S. Wang et al., "Advanced pneumonic-type lung carcinoma: a retrospective study of clinical radiological pathological characteristics with survival analysis in a single Chinese hospital," Zhongguo Fei Ai Za Zhi, vol. 22, no. 6, pp. 329-335, 2019.

[29] W. L. Wang, F. Shen, X. J. Yao, and B. Qian, "Diagnostic value of multi-slice spiral CT on differential diagnosis of peripheral lung cancer and focal machine pneumonia," Computerized Tomography Theory and Applications, vol. 27, no. 4, pp. 529-536, 2018.

[30] O. Stephen, M. Sain, U. J. Maduh, and D.-U. Jeong, "An efficient deep learning approach to pneumonia classification in healthcare," Journal of Healthcare Engineering, vol. 2019, Article ID 4180949, 7 pages, 2019.

[31] C. Liu, X. Wang, C. Liu, Q. Sun, and W. Peng, "Differentiating novel coronavirus pneumonia from general pneumonia based on machine learning," BioMedical Engineering Online, vol. 19, no. 1, p. 66, 2020.

[32] S. H. Kassania, P. H. Kassanib, M. J. Wesolowskic, K. A. Schneidera, and R. Detersa, "Automatic detection of coronavirus disease (COVID-19) in X-ray and CT images: a machine learning based approach," Biocybernetics and Biomedical Engineering, vol. 41, no. 3, pp. 867-879, 2021.

[33] K. El Asnaoui, Y. Chawki, and A. Idri, "Automated methods for detection and classification pneumonia based on X-ray images using deep learning," in Artificial Intelligence and Blockchain for Future Cybersecurity Applications. Studies in Big Data, Y. Maleh, Y. Baddi, M. Alazab, L. Tawalbeh, and I. Romdhani, Eds., vol. 90, pp. 257-284, Springer, Cham, Switcherland, 2021.

[34] Y. Muhammad, M. D. Alshehri, W. M. Alenazy, T. Vinh Hoang, and R. Alturki, "Identification of pneumonia disease applying an intelligent computational framework based on deep learning and machine learning techniques," Mobile Information Systems, vol. 2021, Article ID 9989237, 20 pages, 2021.

[35] Q. Tong, M. Ning, W. Si, X. Liao, and J. Qin, “3D deeplysupervised U-net based whole heart segmentation," in Proceedings of the International Workshop on Statistical Atlases and Computational Models of the Heart, Granada, Spain, September 2018. 
[36] S. Jia, A. Despinasse, Z. Wang et al., "Automatically segmenting the left atrium from cardiac images using successive 3D U-nets and a contour loss," in Proceedings of the Statistical Atlases and Computational Modeling of the Heart (STACOM) workshop, Granada, Spain, September 2018.

[37] T. Wang, J. Xiong, X. Xu et al., "MSU-net: multiscale statistical U-net for real-time 3D cardiac MRI video segmentation," in Proceedings of the 22nd International Conference on Medical Image Computing and Computer Assisted Intervention, Shenzhen, China, October 2019.

[38] J. Wu, Y. Zhang, and X. Tang, "Simultaneous tissue classification and lateral ventricle segmentation via a $2 \mathrm{D} U$-net driven by a 3D fully convolutional neural network," in Proceedings of the 2019 41st Annual International Conference of the IEEE Engineering in Medicine and Biology Society (EMBC), pp. 5928-5931, IEEE, Berlin, Germany, July 2019.

[39] M. Raghu, C. Zhang, J. Kleinberg, and S. Bengio, "Transfusion: understanding transfer learning for medical imaging," in Proceedings of the 33rd Conference on Neural Information Processing Systems (NeurIPS 2019), Vancouver, Canada, December 2019.

[40] S. Guo and Z. Yang, "Multi-Channel-ResNet: an integration framework towards skin lesion analysis," Informatics in Medicine Unlocked, vol. 12, pp. 67-74, 2018.

[41] V. Alex, M. Khened, S. Ayyachamy, and G. Krishnamoorthi, "Medical image retrieval using Resnet-18 for clinical diagnosis," in Proceedings of the Imaging Informatics for Healthcare, Research, and Applications, San Diego, CA, USA, March 2019.

[42] H. Xia, M. Ma, H. Li, and S. Song, "MC-Net: multi-scale context-attention network for medical CT image segmentation," Applied Intelligence, vol. 52, no. 1, pp. 1-12, 2021.

[43] J. Hu, H. Wang, J. Wang, Y. Wang, F. He, and J. Zhang, "SANet: a scale-attention network for medical image segmentation," PLoS One, vol. 16, no. 4, Article ID e0247388, 2021.

[44] W. Fang and X. H. Han, "Spatial and channel attention modulated network for medical image segmentation," in Proceedings of the Asian Conference on Computer Vision, Kyoto, Japan, December 2020.

[45] J. Schlemper, O. Oktay, M. Schaap et al., "Attention gated networks: learning to leverage salient regions in medical images," Medical Image Analysis, vol. 53, pp. 197-207, 2019.

[46] A. Dosovitskiy, L. Beyer, A. Kolesnikov et al., "An image is worth 16x16 words: transformers for image recognition at scale," in Proceedings of the International Conference on Learning Representations. ICLR 2021, Vienna, Austria, May 2021. 